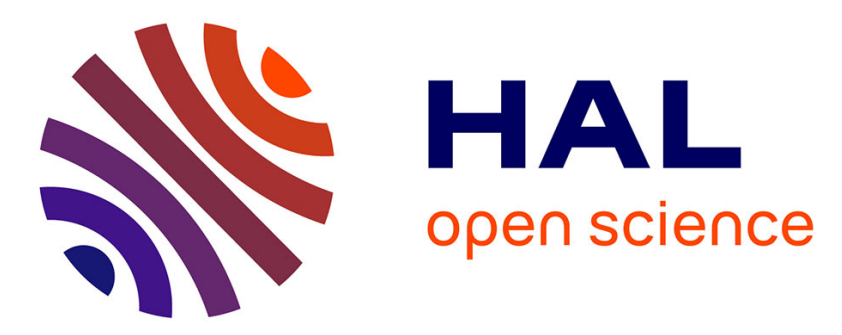

\title{
Determination of the total nuclear spin in a rovibronic state application to the molecules SF6 and PF5
}

\author{
J. Bordé
}

\section{To cite this version:}

J. Bordé. Determination of the total nuclear spin in a rovibronic state application to the molecules SF6 and PF5. Journal de Physique Lettres, 1978, 39 (12), pp.175-178. 10.1051/jphyslet:019780039012017500 . jpa-00231472

HAL Id: jpa-00231472

https://hal.science/jpa-00231472

Submitted on 1 Jan 1978

HAL is a multi-disciplinary open access archive for the deposit and dissemination of scientific research documents, whether they are published or not. The documents may come from teaching and research institutions in France or abroad, or from public or private research centers.
L'archive ouverte pluridisciplinaire HAL, est destinée au dépôt et à la diffusion de documents scientifiques de niveau recherche, publiés ou non, émanant des établissements d'enseignement et de recherche français ou étrangers, des laboratoires publics ou privés. 


\title{
LE JOURNAL DE PHYSIQUE-LETTRES
}

\author{
Classification \\ Physics Abstracts \\ 33.10
}

\section{DETERMINATION OF THE TOTAL NUCLEAR SPIN IN A ROVIBRONIC STATE APPLICATION TO THE MOLECULES SF 6 AND PF $_{5}$}

\author{
J. BORDÉ \\ Laboratoire de Physique Moléculaire et d'Optique Atmosphérique $\left({ }^{*}\right)$, \\ C.N.R.S., Bâtiment 221, Université Paris-Sud, 91405 Orsay Cedex, France
}

(Reçu le 16 février 1978, révisé le 10 avril 1978, accepté le 24 avril 1978)

\begin{abstract}
Résumé. - Pour calculer la structure hyperfine magnétique des raies de vibration-rotation, il faut répondre d'abord à la question suivante : étant donné un état rovibronique, quelles sont les valeurs du spin nucléaire total qui peuvent lui être associées ? Nous donnons trois approches pour résoudre ce problème et nous présentons les résultats pour $\mathrm{PF}_{5}$ et $\mathrm{SF}_{6}$.

Abstract. - In calculating the hyperfine magnetic structure of vibration-rotation lines, one question arises first : which values of the total nuclear spin are allowed in a given rovibronic state ? We present three approaches to solve this problem and the application to $\mathrm{SF}_{6}$ and $\mathrm{PF}_{5}$ is worked out.
\end{abstract}

We consider a molecule having $n$ identical nuclei each nucleus having a spin $i$. It has been established for a long time [1] in Molecular Spectroscopy how one should calculate the statistical weights : one determines the types of symmetry of the nuclear spin functions in the point group of the molecule. Recently, saturated absorption infrared spectra of $\mathrm{CH}_{4}$ and $\mathrm{SF}_{6}$ have been reported [2,3], showing the hyperfine structure of vibration-rotation lines. This structure is due to the spin-spin and spin-rotation magnetic couplings, and in order to calculate these interactions, one must know, in addition to the symmetry types, the values of the total nuclear spin.

More precisely, given a rovibronic state $\Psi_{\text {evr }}$ (which may belong to a degenerate level), the complete wave function must obey the Pauli principle and as a result only a subspace of the $(2 i+1)^{n}$ independant nuclear spin functions $\Phi_{j}$ may be used to construct the complete wave function. As a consequence, among all the functions which correspond to the possible values for the total nuclear spin $I$ and its projection $M_{I}$, some are eliminated and cannot be associated with the space function $\Psi_{\text {evr }}$. We shall present methods which allow us to determine which values of $I$ and $M_{I}$ must be retained.

$\left(^{*}\right)$ This work was supported in part by Université Pierre-etMarie-Curie (Paris-VI).
The result is only known for very simple molecules (the diatomics) or for some molecules for which the magnetic structure has already been studied in the fundamental vibronic state : $\mathrm{NH}_{3}$ [4], $\mathrm{CH}_{4}$ [5]. For these two molecules the result is simple : there is a one-to-one correspondance between the value of the total spin and the symmetry type (which is not the case for $\mathrm{SF}_{6}$ nor $\mathrm{PF}_{5}$ ) and no general method was needed. In reference [5], the particular partitioning method followed by the authors has also been applied to $\mathrm{CD}_{4}$ for which a one-to-one correspondance is not valid and, in reference [12], the case of $\mathrm{C}_{2} \mathrm{H}_{4}$ is studied but with a method which is not easily extended to molecules such as $\mathrm{SF}_{6}$.

We shall use $G$ to designate the point group of the molecule and $D^{(\mu)}$ its irreducible representations (I.R.). $\Phi_{\beta}^{(\mu), \rho}$ will designate functions which are combinations of the $\Phi_{j}$ and which are basis for the I.R. $D^{(\mu)} ; \beta$ numbers the components in the basis of a I.R. $D^{(\mu)}\left(\beta=1, \ldots, d_{\mu}\right.$ if $d_{\mu}$ is the dimension of the I.R. $\left.D^{(\mu)}\right), \rho$ distinguishes different functions with the same $(\mu)$ and the same $\beta\left(\rho=1, \ldots, r_{(\mu)}\right.$ if the I.R. $D^{(\mu)}$ can be constructed $r_{(\mu)}$ times with the $\Phi_{j}$ ). We shall say that the $\Phi_{\beta}^{(\mu), \rho}$ are obtained by symmetry adapting the nuclear spin functions $\Phi_{j}$ in the group $G$. Similarly, we shall denote by $S_{n}$ the group of permutation of $n$ symbols, $D^{\{\lambda\}}$ its I.R. ( $\lambda$ represents a Young diagram $\left.\lambda_{1}, \lambda_{2}, \ldots, \lambda_{n}\right)$, and $\Phi_{\alpha}^{\{\lambda\}, \tau}$ are the nuclear spin functions symmetry adapted in $S_{n}$. 
In the rotation group $\mathrm{SO}(3)$, the I.R. correspond to the values of the total spin $I$, the values of its projection, $M_{I}$, number the components of the I.R. basis and $\Phi_{M_{I}}^{(I), \sigma}$ are the symmetry adapted spin functions.

Let us recall first some essential results deduced from standard group theory $[6,7]$ : the $(2 i+1)^{n}$ functions $\Phi_{j}$ form a basis for a reducible representation of the rotation group $\mathrm{SO}(3)$; this representation, which will be noted $D^{(\mathbf{S})}$, is the $n$-th power of the I.R. $D^{(I)}(I=i)$ of $\mathrm{SO}(3)$. Because of the commutation properties between the operations of the rotation group and the permutations of $\mathrm{S}_{n}$, the matrices of the representation $D^{(\mathbf{S})}$, when expressed in the symmetrized basis $\Phi_{\alpha}^{\{\lambda\}, \tau}$, have the following very simple structure :

$$
D_{\{\lambda\}, \tau, \alpha ;\left\{\lambda^{\prime}\right\}, \tau^{\prime}, \alpha^{\prime}}^{(\mathbf{S})}=\delta_{\{\lambda\}\left\{\lambda^{\prime}\right\}} \delta_{\alpha \alpha^{\prime}} M_{\tau \tau^{\prime}}^{\{\lambda\}} .
$$

In this basis, the representation $D^{(\mathbf{S})}$ of $\mathrm{SO}(3)$ is thus decomposed in blocks, $M_{\tau \tau^{\prime}}^{\{\lambda\}}$; each block is of dimension $r_{\{\lambda\}}, r_{\{\lambda\}}$ being the number of times the I.R. $D^{\{\lambda\}}$ occurs. Each of these blocks may be decomposed as a sum of I.R. of $\mathrm{SO}(3)$; these decompositions lead to a new basis of spin functions $\Phi_{\alpha, M_{I}}^{\{\lambda\},(I), \xi}$. Each of these functions has a definite type of symmetry in the group $\mathrm{S}_{n}$ and corresponds to a definite value for the total nuclear spin and its projection. This allows us to make a correspondance between some of the possible values for the total nuclear spin $I$ and each I.R. $D^{\{\lambda\}}$ of $S_{n}$. We must emphasize that if a value of $I$ is associated with an I.R. $D^{\{\lambda\}}$, it means that the I.R. $D^{(I)}$ of $\mathrm{SO}(3)$ is contained in the representation $M^{\{\lambda\}}$ and so all the values of $M_{I}$, $M_{I}=-I, \ldots,+I$, will be associated with $D^{\{\lambda\}}$.

In the preceding paragraph, we have considered first the group $S_{n}$ and secondly the group $\mathrm{SO}(3)$ in the construction of the functions $\Phi_{\alpha, M_{I}}^{\{\lambda\},(I), \xi}$ which are symmetry adapted in both groups. There is obviously a second way to construct them, by considering the two groups in the inverse order, first $\mathrm{SO}(3)$ secondly $S_{n}$ : the $(2 i+1)^{n}$ functions $\Phi_{M_{I}}^{(I), \sigma}$ form a basis for a reducible representation of $S_{n}$; the matrices of this representation have non-zero off-diagonal elements only if the two basis functions have identical $I$ and $M_{I}$. These blocks, of dimension $r_{(I)}$, will then be decomposed as sums of I.R. of $\mathrm{S}_{n}, D^{\{\lambda\}}$ (or as sums of I.R. of any subgroup of $\mathrm{S}_{n}$ ). We shall present three methods to obtain the results we look for ; the first two methods are deduced from the first way of constructing the $\Phi_{\alpha, M_{I}}^{\{\lambda\},(I), \xi}\left(\mathrm{S}_{n}\right.$ then $\left.\mathrm{SO}(3)\right)$, the third method is based on the second way $(\mathrm{SO}(3)$ then $S_{n}$ ).

In the case where $i=\frac{1}{2}$, a great simplification occurs : only one value of $I$ is associated with each $D^{\{\lambda\}}$ : it can be shown that, first, the only I.R. of $S_{n}$ appearing in the reduction of $D^{(\mathbf{S})}$ are characterized by the Young diagrams such that : $\{\lambda\}=\left\{\lambda_{1}, \lambda_{2}, 0\right.$, $0, \ldots 0\}$ and, second, that the blocks $M_{\tau \tau^{\prime}}^{\{\lambda\}}$ form the
I.R. of $\operatorname{SO}(3)$ corresponding to $I=\frac{\lambda_{1}-\lambda_{2}}{2}$. When

$i>\frac{1}{2}$, one must decompose the matrices $M_{\tau \tau^{\prime}}^{\{\lambda\}}$ as sums of I.R. of $\mathrm{SO}(3)$. In order to do this, it is possible either to calculate the characters of these matrices [6], or to follow an iteration method [7].

To apply all this to a molecule, one must introduce the fact that the point group $G$ is in general isomorphic not to $S_{n}$ but to a subgroup of $S_{n}^{*}=S_{n} \times C_{i}$, where $C_{i}$ is the two-elements group constitued by identity and inversion $\left(^{1}\right)$. Each I.R. of $\mathrm{S}_{n}^{*}, D^{\{\lambda\} \mathrm{p}}$ $(\mathrm{p}=\mathrm{g}$ or $\mathrm{u})$, supplies a reducible representation of $\mathrm{G}$ which can be decomposed on the I.R. $D^{(\mu)}$ of G. Conversely, each I.R. $D^{(\mu)}$ of $\mathrm{G}$ induces, by extending if needed its space of representation, a representation of $\mathrm{S}_{n}^{*}$ which can be decomposed on the I.R. $D^{\{\lambda\} \text { p }}$. According to Frobenius' theorem, if the I.R. $D^{(\mu)}$ induces the representation $\sum_{\{\lambda\} \mathbf{p}} a_{\{\lambda\} \mathbf{p}}$ $D^{\{\lambda\} \mathrm{p}}$, it implies that the representation of $\mathrm{G}$ supplied by the matrices $D^{\{\lambda\} \text { p }}$ contains $a_{\{\lambda\}}$ times the I.R. $D^{(\mu)}$ of $\mathrm{G}$. In particular, the representation of $\mathrm{S}_{n}^{*}$ which satisfies the Pauli principle, being of dimension 1, supplies only one I.R. of G (the I.R. which satisfies the Pauli principle in G) and, according to Frobenius, can be induced only by this I.R. of G. This gives a proof that the statistical weights obtained from any subgroup of $S_{n}^{*}$ are identical to those one would obtain from $\mathrm{S}_{n}^{*}$.

From all the previous considerations, we can state that three methods are possible to determine which values of $I$ may be associated with a rovibronic state $\Psi_{\mathrm{evr}}^{(\mu)}$ belonging to the symmetry type $D^{(\mu)}$ :

1st METHOD. - a) One determines which I.R. $D^{\{\lambda\} \mathrm{p}}$ of $S_{n}^{*}$ are induced by the I.R. $D^{(\mu)}$ of $\mathrm{G}$;

$b)$ from $a$ ) one deduces which symmetry types $D^{\left\{\lambda^{\prime}\right\} p^{\prime}}$ the nuclear spin functions must belong to, so that the complete wavefunction obeys the Pauli principle ;

c) from the correspondance between the values of $I$ and each $D^{\left\{\lambda^{\prime}\right\} \mathbf{p}^{\prime}}$, one finds the values of $I$ for the state $\Psi_{\mathrm{evr}}^{(\mu)}$.

2nd METHOD. - We can restrict the use of $S_{n}^{*}$ to the spin functions :

a) one determines the I.R. $D^{\left(\mu^{\prime}\right)}$ of $\mathrm{G}$ to which the spin functions must belong so that the Pauli principle is satisfied ;

b) one determines the I.R. $D^{\left\{\lambda^{\prime}\right\} \mathbf{p}^{\prime}}$ induced by $D^{\left(\mu^{\prime}\right)}$;

c) as in $1 c)$.

3rd METHOD. - We can avoid introducing the group $S_{n}^{*}$ and work only in the point group $G$. The principle of this method is also to construct doubly

$\left({ }^{1}\right)$ In the inversion of the space fixed axes, the spin functions are gerade and the previous results of group theory can immediately by extended from the two groups $\mathrm{S}_{n}$ and $\mathrm{SO}(3)$ to the two groups $\mathrm{S}_{n}^{*}$ and $\mathrm{O}(3)$. 
symmetry adapted spin functions $\Phi_{M_{I}, \beta}^{(I),(\mu), \xi}$ but, this time, in $O(3)$ and in the point group $G$ [8] :

a) for each possible value $I$ of the total spin, we calculate explicitly the $r_{(I)}$ functions corresponding to an arbitrarily chosen value of $M_{I}$ (it may be easier to take $\left.M_{I}=I\right)$. These functions can be expressed as :

$$
\Phi_{M_{I}}^{(I), \sigma}=\sum \alpha_{m_{1}, m_{2}, \ldots m_{n}}^{(I), \sigma, M_{I}} \varphi_{m_{1}}^{(i)} \varphi_{m_{2}}^{(i)} \ldots \varphi_{m_{n}}^{(i)}
$$

where $\varphi_{m_{j}}^{(i)}$ is the elementary spin function of the $j^{\text {th }}$ nucleus. The coefficients $\alpha_{m_{1}, \ldots, m_{n}}^{(I), \sigma, M_{I}}$ can be obtained from the Clebsch-Gordan coefficients ;

$b$ ) for each operation $R$ of the point group $G$ and each value of $I$, we can obtain the character $\chi_{R}^{(I)}$ of the matrix $M_{\sigma \sigma^{\prime}}^{(I)}$ of the representation of $\mathrm{G}$ generated by the $r_{(I)}$ functions $\Phi_{M_{I}}^{(I), \sigma}:$ if the operation $R$ corresponds to the permutation $\left(\begin{array}{cccc}p_{1}, & p_{2}, & \ldots, & p_{n} \\ 1, & 2, & \ldots, & n\end{array}\right)$, we have :

$$
\chi_{R}^{(I)}=\sum_{m_{1}, m_{2}, \ldots, m_{n}} \alpha_{m_{1}, m_{2}, \ldots, m_{n}}^{(I), \sigma, M_{I}} \alpha_{m_{p_{1}}, m_{p_{2}}, \ldots, m_{p_{n}}}^{(I), \sigma, M_{I}} ;
$$

c) by decomposing the representations $M^{(I)}$ on the I.R. $D^{(\mu)}$ of $\mathrm{G}$, one obtains the symmetry types associated with each value of $I$. Each of these decompositions is in fact obtained $2 I+1$ times (for each value of $M_{I}$ ).

According to the case, one of the three methods will appear easier or more appropriate to other problems one wants to solve with the spin functions.

Application to $\mathbf{S F}_{6}$ and $\mathbf{P F}_{5}$. - For these two molecules $i=\frac{1}{2}, n$ is not too large and the three methods can be used. Because of the simplification due to $i=\frac{1}{2}$ and because the characters of the I.R. are well-known for $S_{5}$ and $S_{6}$, we can find at once the character $\chi_{R}^{(I)}$ :

- for $\mathrm{PF}_{5}$, the possible values for the total spin are $I=5 / 2,3 / 2,1 / 2$; they correspond respectively to the I.R. of $S_{5}^{*} D^{\{5\} \mathrm{g}}, D^{\{4,1\} \mathrm{g}}$ and $D^{\{3,2\} \mathrm{g}}$; we know the characters of these I.R. of $S_{5}^{*}$ so that it is easy to decompose them in $\mathrm{D}_{3 \mathrm{~h}}$;

- for $\mathrm{SF}_{6}$, the values of $I$ are $3,2,1,0$ and corres-

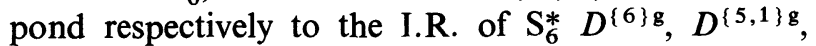
$D^{\{4,2\} \mathrm{g}}$ and $D^{\{3,3\} \mathrm{g}}$. One only has to decompose these representations on the I.R. of the point group $\mathrm{O}_{h}$ of $\mathrm{SF}_{6}$.

We have recalculated these values of $\chi_{R}^{(I)}$ just as if the simplification due to $i=\frac{1}{2}$ did not exist and as if the characters of the I.R. of $S_{n}^{*}(n=5,6)$ were not known. To do so, we have written a computer programme which executes automatically the two first steps of the third method and calculates the $\chi_{R}^{(I)}$ using the equation (3). This programme may be used for molecules with nuclei of spin greater than $\frac{1}{2}$ and for molecules with larger $n$, for which it is more difficult to handle the group $\mathrm{S}_{n}^{*}$ and work out the two first methods.

The values of the characters $\chi_{R}^{(I)}$ and the decompositions in the group $\mathrm{G}$ are given in table $\mathrm{I}$ for $\mathrm{SF}_{6}$ and in table II for $\mathrm{PF}_{5}$. Multiplying each decomposition by $2 I+1$ leads to the total number of each I.R. of $\mathrm{G}$ which may be constructed with the nuclear spin functions. In order to associate values of $I$ with the spatial function $\Psi_{\text {evr }}^{(\mu)}$, one needs to know the correspondance between $D^{(\mu)}$ and the I.R. $D^{\left(\mu^{\prime}\right)}$ such that the $\Psi_{\text {evr }}^{(\mu)} \Phi^{\left(\mu^{\prime}\right)}$ obey the Pauli principle. This correspondance is given in the published works on statistical weights (see [9] for $\mathrm{PF}_{5}$ and $[10,11]$ for $\mathrm{SF}_{6}$ ) and we shall not recall it here.

\section{TABLE I}

Correspondance between the values of the total nuclear spin

\begin{tabular}{|c|c|c|c|c|c|c|c|c|c|c|c|c|c|}
\hline & \multicolumn{10}{|c|}{$\begin{array}{l}\text { Classes of the operations }(*) \\
\text { of the point group } O_{h}\end{array}$} & & \\
\hline & & $\begin{array}{l}E \\
1^{6}\end{array}$ & $\begin{array}{l}8 C_{3} \\
3^{2}\end{array}$ & $\begin{array}{c}6 \mathrm{C}_{2} \\
2^{3}\end{array}$ & $\begin{array}{l}6 C_{4} \\
1^{2} 4^{1}\end{array}$ & $\begin{array}{l}3 C_{4}^{2} \\
1^{2} 2^{2}\end{array}$ & $\begin{array}{c}\mathrm{i} \\
2^{3}\end{array}$ & $\begin{array}{r}6 S_{4} \\
2^{1} 4^{1}\end{array}$ & $\begin{array}{c}8 \mathrm{~S}_{6} \\
6\end{array}$ & $\begin{array}{l}3 \sigma_{\mathrm{h}} \\
1^{4} 2^{1}\end{array}$ & $\begin{array}{l}6 \sigma_{\mathrm{d}} \\
1^{2} 2^{2}\end{array}$ & & \\
\hline \multirow{4}{*}{$\chi_{R}^{(I)}$} & $I=3$ & 1 & 1 & 1 & 1 & 1 & 1 & 1 & 1 & 1 & 1 & $A_{1 g}$ & \multirow{4}{*}{ 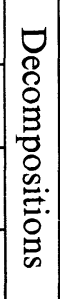 } \\
\hline & $I=2$ & 5 & -1 & -1 & 1 & 1 & -1 & -1 & -1 & 3 & 1 & $E_{g}+F_{1 u}$ & \\
\hline & $I=1$ & 9 & 0 & 3 & -1 & 1 & 3 & 1 & 0 & 3 & 1 & $A_{1 g}+E_{g}+F_{2 g}+F_{2 u}$ & \\
\hline & $I=0$ & 5 & 2 & -3 & -1 & 1 & -3 & -1 & 0 & 1 & 1 & $A_{2 g}+A_{2 u}+F_{1 u}$ & \\
\hline
\end{tabular}
and the symmetry types of the spin functions for $\mathrm{SF}_{6}$

$\left.{ }^{*}\right)$ The cycles structure of the permutation operating on the indices of the spins is given under the geometrical notation. 
As a sample application of our tables, let us take two examples for $\mathrm{SF}_{6}$ :

a) given a rovibronic level of type $\mathrm{A}_{2 \mathrm{~g}}$; the spin function must be of type $A_{1 g}$ or $A_{1 u}$. It is only possible to construct 10 spin functions of type $A_{1 \mathrm{~g}}$ and which correspond respectively to the spin quantum numbers :

$$
\begin{array}{ll}
I=3, M_{I}=-3 ; & I=3, M_{I}=-2 ; \\
I=3, M_{I}=-1 ; & I=3, M_{I}=0 ; \\
I=3, M_{I}=1 ; & I=3, M_{I}=2 ; \\
I=3, M_{I}=3 ; & I=1, M_{I}=-1 ; \\
I=1, M_{I}=0 ; & I=1, M_{I}=1 ;
\end{array}
$$

b) given a rovibronic level of type $\mathrm{F}_{2 \mathrm{~g}}$; the spin functions must be of type $F_{1 u}$ or $F_{1 \mathrm{~g}}$. It is only possible to construct six basis of type $F_{1 u}$. Each basis is composed of three functions, each of these three functions may be taken as an eigenfunction of $I^{2}$ and $I_{z}$ with the same value of $I$ and $M_{I}$ for all three. The six basis correspond respectively to the following values of $I$ and $M_{I}$ :

$$
\begin{array}{ll}
I=2, M_{I}=-2 ; & I=2, M_{I}=-1 \\
I=2, M_{I}=0 ; & I=2, M_{I}=-1 \\
I=2, M_{I}=2 ; & I=0, M_{I}=0 .
\end{array}
$$

Finally, we shall give an example of application of method 1 for a level $A_{1}^{\prime}$ of $P_{5}$ so that the reader be convinced that different methods give the same result.

In $S_{5}^{*}$, symmetry adapted nuclear spin functions are of type $D^{\{5\} \mathrm{g}}, D^{\{4,1\} \mathrm{g}}$ and $D^{\{3,2\} \mathrm{g}}$ and symmetry adapted vibration-rotation functions induced by a $A_{1}^{\prime}$ function are $D^{\{5\} \mathrm{g}}, D^{\{4,1\} \mathrm{g}}, D^{\{3,2\} \mathrm{g}}, D^{\left\{1^{5}\right\} \mathrm{u}}, D^{\left\{2,1^{3}\right\} \mathrm{u}}, D^{\left\{2^{2}, 1\right\} \mathrm{u}}$. According to Pauli, these space functions may be associated with the spin functions $D^{\{5\}}$ (associated with $D^{\left\{1^{5}\right\} \text { u }}$ ), $D^{\{4,1\} g}$ (associated with $D^{\left\{2,1^{3}\right\} \text { u }}$ ) and $D^{\{5,2\} g}$ (associated with $D^{\left\{2^{2}, 1\right\} \text { u }}$ ).

To these symmetry adapted spin functions correspond respectively the values of $I, 5 / 2,3 / 2$ and $1 / 2$ and so these three values of the total nuclear spin exist for a $A_{1}^{\prime}$ vibration-rotation level. The same conclusion may be read in table II since, according to Pauli, the spin functions must be $A_{1}^{\prime}$ or $A_{1}^{\prime \prime}$ in $D_{3 h}$ for a $A_{1}^{\prime}$ vibration-rotation level.

\section{TABLE II}

Correspondance between the values of the total nuclear spin I and the symmetry types of the spin functions for $\mathrm{PF}_{5}$

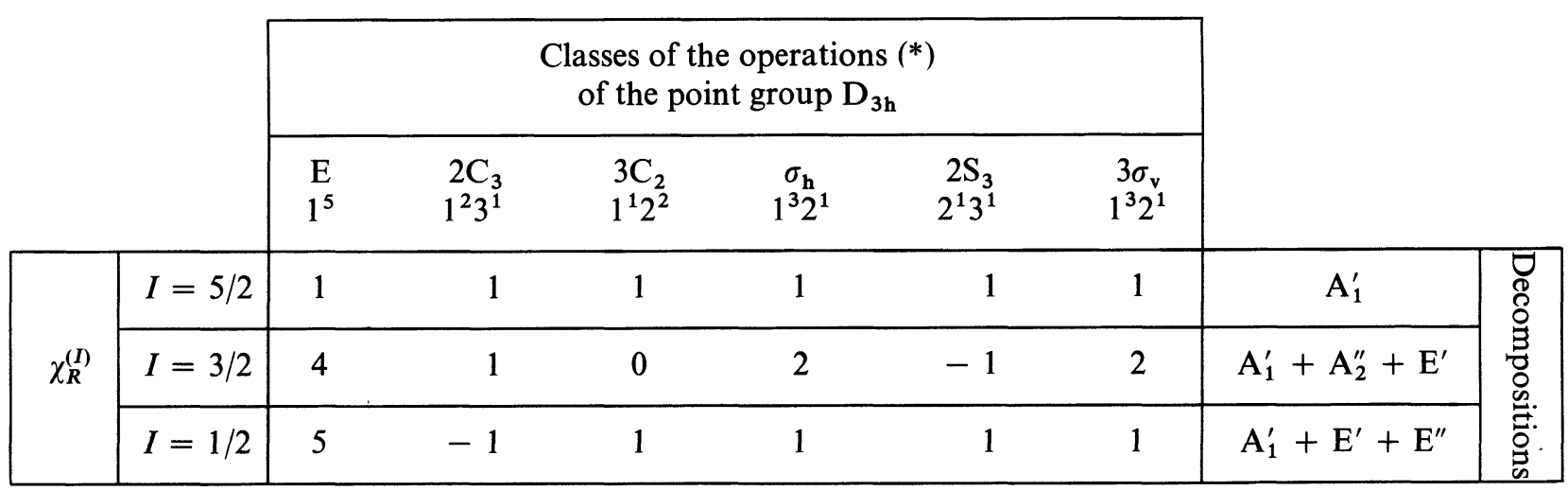

$\left(^{*}\right)$ The cycles structure of the permutation operating on the indices of the spins is given under the geometrical notation.

\section{References}

[1] Wilson, E. B. Jr., J. Chem. Phys. 3 (1935) 276.

[2] Hall, J. L., Bordé, C. J., Uehara, K., Phys. Rev. Lett. 37 (1976) 1339.

[3] Avrillier, S., Vanlerberghe, A., Ouhayoun, M., Bordé, C. J., 5th Colloquium on High Resolution Molecular Spectroscopy, Tours 1977.

[4] Gunther-Mohr, G. R., Townes, C. H., van Vleck, J. H., Phys. Rev. 94 (1954) 1191.

[5] Pon-Nyong Yi, Ozier, I., Anderson, C. H., Phys. Rev. 165 (1968) 92.

[6] Pétrachène, M., Trifonov, E., Applications de la théorie des groupes à la Mécanique Quantique (Masson et Cie, France), 1970.
[7] HAMERMESH, M., Group theory and its applications to physical problems (Addison-Wesley Company, U.S.A.), 1962.

[8] Cederberg, J. W., Am. J. Phys. 40 (1972) 159.

[9] Dalton, B. J., J. Chem. Phys. 54 (1971) 4745 ;

Chéron, M., Thèse de 3e Cycle, Paris VI, 1973.

[10] Cantrell, C. D., Galbraith, H. W., J. Molec. Spectrosc. 58 (1975) 158.

[11] Berger, H., J. Physique 38 (1977) 1371.

[12] LandaU, L., Lifchitz, E., Mécanique Quantique (Editions MIR), 1967, p. 467. 